\title{
Elastic full-field strain analysis and microdamage progression in the vertebral body from digital volume correlation
}

\author{
Gianluca Tozzi ${ }^{a^{*}}$, Valentina Danesi ${ }^{\mathrm{b}}$, Marco Palanca ${ }^{\mathrm{b}}$, Luca Cristofolini ${ }^{\mathrm{b}}$

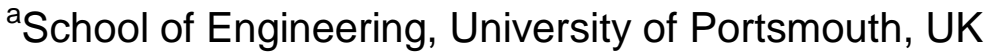 \\ ${ }^{\mathrm{b}}$ Department of Industrial Engineering, Alma Mater Studiorum - University of \\ Bologna, Italy
}

Address for correspondence:

Dr Gianluca Tozzi

School of Engineering

Anglesea Building, Anglesea Road

Portsmouth

PO1 3DJ

United Kingdom

Tel: +44 (0)239284 2514

Email: gianluca.tozzi@port.ac.uk 


\section{ABSTRACT}

The strain distribution in vertebral body has been measured in vitro in the elastic regime, but only on the bone surface by means of strain gauges and digital image correlation. Digital volume correlation (DVC) based on micro-CT images allowed measurements of the internal strain distribution in bone at both tissue (trabecular and cortical bone) and organ (vertebra) level. However, DVC has been mainly used to investigate failure of the vertebral body, but has not yet been deployed to investigate the internal strain distribution in the elastic regime. The aim of this study was to investigate strain in the elastic regime and up to failure inside the vertebral body, including analysis of strain in all directions. Three porcine thoracic vertebrae were loaded in a step-wise fashion at increasing steps of compression $(5 \%, 10 \%, 15 \%)$. Micro-CT images were acquired at each step of compression. DVC successfully provided the internal strain distribution both in the elastic regime and up to failure. Micro-CT images successfully identified regions of failure initiation and progression, which were well quantified by DVC-computed strains. Interestingly, the same regions where failure eventually occurred experienced the largest strain magnitude also for the lowest degrees of compression (yet in the elastic regime).

Keywords: bone, digital volume correlation, elastic strain, micro-CT, vertebral body. 


\section{Introduction}

Pathologies such as osteoporosis and bone metastases are the major causes of vertebral fractures, often in combination with trauma or para-physiological overloading. These vertebrae are weak because their micro- and/or macro-structure are pathologically compromised. If untreated, they might fracture, causing severe disabilities and in some cases even mortality [1, 2]. For this reason, knowledge of the failure mechanism in the vertebra is of fundamental importance to understand vertebral biomechanics [3], improve diagnosis and prophylactic treatments $[4,5]$.

In vitro testing of the vertebral body has been extensively carried out in the past [6-8]. The strain distribution in the vertebral body was investigated using different experimental techniques but mainly with strain gauges [9], where the fullfield strain distribution was not investigated. Furthermore, strain gauges are associated with a reinforcement effect that in the case of a thin shell of cortical bone cannot be neglected [10-12].

More recently, digital image correlation (DIC) was adopted to investigate the full-field strain distribution on the cortical surface of vertebrae, in an attempt to avoid direct contact measurement (i.e. via strain gauges) that could potentially produce important artifacts (i.e. reinforcement effect) in the local strain determination [13]. To this extent, [14] presented a comparison of strain rosettes and DIC to measure the vertebral body strain. In that study porcine vertebrae were prepared with a strain rosette plus a speckled paint pattern for DIC and loaded in compression. However, it must be pointed out that also the specimen preparation for an appropriate DIC measurement (i.e. speckle pattern distribution) must be planned carefully if reliable results are to be achieved $[13,15]$.

When measuring strain in bone in vitro, one must consider the magnitude of strain experienced during physiological tasks in vivo (1000-2000 microstrain, [16, 17]). Moreover, bone typically exhibits a linear-elastic behaviour up to the yield point in compression $[18,19]$. For the human vertebral trabecular bone, the first evidence of yielding begins at strains of approximately 8000 microstrain, and macroscopic failure begins at strains of the order of 15000 microstrain [20]. The 0.2\%-offset strain of the femoral trabecular bone in compression is $10400+/-1500$ microstrain, [21]). 
The overall precision that can be obtained with strain gauges when applied to bone is of the order of $1-2 \%$ of the readout $[10,22]$. This corresponds to $10-20$ microstrain when the in vitro loads aim to replicate physiological loading scenarios [23], with in vitro strains of the order of 1000 microstrain. The overall precision that can be obtained with DIC (which is mainly limited by noise) is of the order of 100-300 microstrain $[10,13,14]$.

The main limitation of strain gauges and DIC is their inability to capture and quantify internal microdamage evolution and full-field strain distribution under load. As the internal trabecular bone of the vertebral body plays a fundamental structural role $[3,18,24]$, it would be extremely important to measure the internal strain distribution. In fact, a number of studies have shown that in several cases failure starts inside the vertebral body itself $[25,26]$. In this perspective, digital volume correlation (DVC) is ideal to investigate the internal strain distribution and the local damage inside the vertebra. In recent years, DVC has become a powerful tool to examine full-field internal deformations mainly in trabecular [27-31] and cortical bone $[29,31,32]$. The use of DVC to investigate the strain distribution in vertebrae has been firstly introduced by [33]. In that study a new image registration algorithm was developed to spatially resolve strain in whole bones (rat vertebrae) using micro-CT images. Since then, a number of studies investigated the full-field strain distribution in vertebral bodies without [34] and with the adjacent intervertebral discs [35], as well as entire vertebrae [36] under compressive loading. In [34] the highest strain magnitudes (minimum principal strain) were distributed in the superior-inferior (axial) direction ranging between -20000 and -40000 microstrain, in human vertebral bodies. In a following study the same Authors compared the strain distribution in the vertebral body (rabbits) with and without the intervertebral discs [35] up to yield and failure. In both studies $[34,35]$ there is no information on the progression of strain in the elastic regime, preceding the final failure event. Also, the influence of strain directionality and local levels of strain on microdamage evolution in the vertebra has not been investigated. Hardisty et al. [36] is the only study to date to report the microdamage in metastatic and healthy vertebrae (rat models) associated with fullfield strain from DVC, but only for the axial strain. That work reported an average axial strain at failure of -27000 microstrain for the healthy group (5 specimens), but no information of the critical strain values in different locations of the vertebrae. 
Another important aspect to be considered is the level of uncertainly of the DVC measurements, which is associated to imaging conditions, bone type, image preparation, computation sub-volume size and nature of the DVC approach (i.e. local vs global). Similarly to DIC, DVC has very small errors on the computed displacements (0.6-1.2 micrometers, when a whole vertebra was investigated [37]). DVC-computed strains suffer larger errors [38]: the accuracy can range between 300 and 794 microstrain, while the precision between 69 and 630 microstrain [30].

Very recently, an in-depth methodological investigation of all those aspects for natural and augmented vertebral bodies (porcine models) was carried out [37, 39]. Those studies reported that strain uncertainties can be reduced below 300 microstrain for both local and global approaches, for this type of specimens and images. To minimize strain uncertainties, the images are adequately prepared (excluding the non-tissue background), and a wide investigation of the DVC parameters was performed before choosing the optimal computation sub-volume size, related to the spatial resolution of the images (i.e. sub-volume of 48 voxels for a 39 micrometers voxel size image).

In this study, full-field strain distributions inside porcine vertebral bodies were obtained thought DVC under compressive load. Specifically, the main aims of this paper were:

1) To measure the internal strain from the elastic regime up to failure;

2) To analyze the distribution of the different components of strain (axial, antero-posterior and lateral-lateral) for each specimen;

3) To identify microdamage initiation/progression during loading, and to compare with the distribution of the three components of strain.

\section{Materials and methods}

\subsection{Specimens}

Three thoracic vertebrae (specimens T1, T2, T3) were harvested from animals that were bred and slaughtered for alimentation purposes. All the surrounding soft tissues were removed, including the ligaments and discs. The vertebrae were obtained from young animals, where the growth plates were still fully 
open. To avoid the presence of soft tissue and prevent viscoelastic phenomena (which might compromise image acquisition under load), the growth plates were removed together with the adjacent endplates (due to the young age of the animals at sacrifice, this could be performed with little manual effort). The cranial and caudal extremities of the vertebrae were aligned and potted in poly-methyl-methacrylate (PMMA) for a depth of about $4 \mathrm{~mm}$ for each side, following a procedure adapted from [40]. The spinous process was used to center the specimen in the transverse plane and align it about its vertical axis. The posterior arch was subsequently removed.

\subsection{Loading and imaging}

Step-wise compression testing of the vertebrae in combination with timelapsed micro-CT imaging was performed. In situ testing was conducted by means of a loading device (CT5000, Deben Ltd, UK), equipped with a 5kN load cell and a custom-designed environmental chamber which was filled with physiological saline solution (Fig. 1). The specimens were constrained against rotation inside the loading device with sandpaper discs applied to the bottom compressive platen. A preload of $50 \mathrm{~N}$ was applied. Each specimen was compressed axially under displacement control in a step-wise fashion (actuator speed: $0.1 \mathrm{~mm} / \mathrm{sec}$ ). The compression steps were adjusted for each specimen based on its free height, so that at each step the actuator moved by $5 \%$ of the free height for each specimen (this corresponded to actuator steps of 0.54-0.66 mm, depending on the size of the specimen). It must be noted that such actuator displacement included the actual bone compression, but also the compression of the PMMA pots, and the compliance of the entire loading system.

Micro-CT imaging (XTH225, Nikon Metrology, UK) was carried out at each step (0\% with $50 \mathrm{~N}$ preload, $5 \%, 10 \%$ and $15 \%$ compression). To reduce the timedependent phenomena during imaging, the specimen was allowed to settle for 15 minutes after each compression step. Most of the relaxation (Fig. 2) occurred within the 15-minute window. Some additional relaxation was unavoidable during imaging, but was one order of magnitude smaller than the initial one (it never exceeded 10\% of the initial force). Temperature inside the micro-CT chamber was not monitored. 
However, the temperature inside this micro-CT model is quite stable (typically ranging $20-22^{\circ} \mathrm{C}$ [42]). Variations in the range $20-37^{\circ} \mathrm{C}$ have little effect on the mechanical properties of cancellous bone [41].

The micro-CT scanner was set to a voltage of 88-89 kV, a current of $115-116$ microA and exposure time of 2 seconds. The image acquisition was performed at a rotational step of $0.23^{\circ}$ over $360^{\circ}$ for a scanning time of approximately $90 \mathrm{~min}$ at each compression step. The reconstructed micro-CT images had an isotropic voxel size of 38.8 micrometers.

\section{[Figure 1]}

\subsection{Digital volume correlation (DVC)}

DaVis DVC software (v8.3, LaVision, Germany) was used to compute the fullfield strains in the vertebra along the axial, antero-posterior and lateral-lateral directions. The operating principle of the DaVis DVC has been detailed elsewhere $[31,43]$. Briefly, DaVis sub-divides the 3D images into smaller sub-volumes that can be correlated independently (local approach) as a discrete function of grey-levels. The matching between the sub-volumes corresponding to the different stages of loading is achieved via a direct correlation function (DaVis-DC). Due to specimen stability within the loading device, absence of relative rotation and translation of the device throughout the test and small displacements applied, no preparatory rigid body registration was performed. Additionally, a piece-wise linear shape function and a third-order spline interpolation in the image reconstruction are employed to help correlation of the pattern information contained in the reference and deformed images. The displacement vector field is obtained at the center of each sub-volume. The strain field is subsequently computed using a centered finite differences (CFD) scheme. The original micro-CT images were masked in order to remove the background areas where no bone was present. In fact, it was shown that regions that do not contain useful feature for the correlation algorithm are associated with large strain artifacts [37, 39]. A user-defined polygon mask was created, which corresponded to the contour shape of each vertebral body. The mask was defined in the transverse plane of the vertebral body and sequentially adapted in the caudal- 
cranial direction to follow the shape of the vertebra. The geometric mask enabled the DVC software to include only the voxels inside the mask (vertebral body area).

The micro-CT scan setting and DVC parameters relied on previous methodological works [37, 39]. Briefly: repeated micro-CT scans of the vertebral body in zero-strain conditions were processed using a wide range of sub-volume sizes. The systematic and random errors were evaluated considering the unavoidable compromise between measurement uncertainties and spatial resolution [37, 39].

The present DVC computation relied on a final sub-volumes of 48 voxels, reached after successive (predictor) passes using sub-volumes of 128 voxels, 112 voxels, 96 voxels, 80 voxels and 64 voxels, with a $0 \%$ overlap. This multipass sequence and the final sub-volume produced the lowest strain error in DaVis-DC, for such type of specimens, with the same imaging and environmental settings as described in $[37,39]$. Errors on the DVC-computed displacements using this multipass scheme do not exceed 1.2 micrometers [37]. Random errors on the DVCcomputed strains do not exceed 65 microstrains, whereas systematic errors between -71 and 118 microstrain were expected [37]. Given the voxel size of the micro-CT images, the final computation sub-volume size corresponded to 1862 micrometers.

In order to evaluate the strain distribution in the vertebra and to couple local high-strains with visible microdamage, dedicated Matlab (v2016, MathWorks, US) scripts were developed. The Matlab script allowed: (i) exposing any slice (frontal, sagittal or transverse) within the volume, and (ii) computing the average strain (axial, antero-posterior and lateral-lateral components of strain) for each transverse section in the caudal-cranial direction. This allowed identifying the most strained level along the vertebral body and the localization of the strain peaks within the frontal and sagittal sections.

\section{Results}

The force-displacement curves (Fig. 2) showed an initial toe region, which depends on the initial co-planarity of the two pots (which is never perfect). After the toe region, all the specimens showed a monotonic trend that was linear until failure. 
The linear region always extended beyond the first compression step (5\%). Failure was clearly visible as a plateau and decrease in the force-displacement plots, and occurred at $10 \%$ or $15 \%$ steps in all specimens (Fig. 2 and Table 1). Relaxation was also visible at the end of each step, when the actuator was stopped to allow microCT scanning.

[Figure 2]

\section{[Table 1]}

The internal strain distributions (axial, antero-posterior and lateral-lateral components of strain) for the three compression steps $(5 \%, 10 \%$ and $15 \%)$ on the sagittal section of the three specimens are reported in Figures 3-5.

The micro-CT images of specimen T1 showed a main microdamage localized in the trabecular bone (caudal region), which started to appear at the $10 \%$ compressive step, and degenerated into a trabecular collapse at 15\% (Fig. 3).

\section{[Figure 3]}

Such a collapse gradually led to a weakening of the vertebral body in the transverse plane, with damage extending to the cortical bone anteriorly. The distribution of the three components of strain well described the damage events, with the maximum strains located in regions adjacent to the crushed zone; away from the crushed region the strains were significantly lower (Fig. 3).

A similar agreement between the damage (visible in the micro-CT images) and the distribution of strain (computed by means of DVC) was found in the other two specimens, although the damage pattern was different (Fig. 4 and 5). In specimen T2 the microdamage seemed to be localized in the trabecular structure as a gradual collapse that initiated (10\%) and then propagated (15\%) posteriorly, along the caudal-cranial direction (Fig. 4), similarly to specimen T1. In specimen T3 damage initiated in the cranial region ( $10 \%$ compression) and progressively extended as a collapse in a transverse plane (15\% compression) (Fig. 5). 
[Figure 4]

In general, for all specimens the increase of strain was larger from $10 \%$ to $15 \%$ compression, than from $5 \%$ to $10 \%$ compression, both for the axial component of strain (compressive), and the antero-posterior and lateral-lateral ones (tensile).

\section{[Figure 5]}

For all specimens, the strain distribution in the elastic regime (first step of loading, 5\%) showed a non-uniform strain distribution, which seemed to predict the location of damage initiation before it actually became identifiable (Fig. 3-5).

The progression of strain (axial, antero-posterior and lateral-lateral components of strain) during compression for the three specimens is shown in Figure 6 in terms of average strain at each transverse section.

[Figure 6]

Specimen T1 experienced the highest axial compressive strain (nearly -76000 microstrain, average over the most strained transverse section), followed by specimen T3 (just above -42000 microstrain) and specimen T2 (nearly -33000 microstrain). For the antero-posterior component of strain, the most strained regions reached the range of 6200-8000 microstrain (average over the most strained transverse section), in all specimens. For the lateral-lateral component of strain, the most strained regions were in the range of 3400-9000 microstrain (average over the most strained transverse section), in all specimens. The strain pattern along the caudal-cranial direction was similar for specimens $\mathrm{T} 1$ and $\mathrm{T} 2$, with the largest deformation localized in correspondence of the first quarter caudal. In specimen T3 the highest axial strain magnitudes were found where the cortical shell was mostly curved (first quarter cranial); the largest antero-posterior and lateral-lateral strains were observed in correspondence of the cranial and caudal endplates. The cranialposterior portion of this specimen was in a compressive state, with the largest strain (exceeding -5300 microstrain) at $15 \%$ loading step. 


\section{Discussion}

The first aim of this paper was to evaluate the internal strain distribution (axial, antero-posterior and lateral-lateral components of strain) from DVC in porcine vertebral body, under applied compressive force. A deeper understanding of the internal elastic full-field strain distribution was achieved. In fact, despite a number of studies used DVC to investigate the vertebral global fracture under compression [3436], the elastic strain distribution is still unexplored. The results clearly showed how local strain built up from the elastic regime, and highlighted those internal weaker regions that could result in microdamage initiation and progression up to vertebral failure (Fig. 3-5). When a compression of 5\% was applied, all specimens experienced levels of internal tensile and compressive strains above or close to the typical values of bone tissue failure (i.e. 7000 microstrain for tensile and -10000 microstrain for compression as reported in [3]). For two specimens (T1 and T2) rather regular strain maps were identified for each component of strain, and for the steps of applied compression. Conversely, the third specimen (T3) exhibited a more irregular strain distribution, possibly associated with the superimposition of compression and some degree of bending.

The benefit of using DVC compared to surface strain measurement techniques (i.e. strain gauges or DIC) is particularly evident in specimen T1. In fact, surface strain measurement in the $5 \%$ compression step (force $=1115 \mathrm{~N}$ ) would have only provided information on the strain distribution on the cortical shell that was mostly below the yield values for bone in both compression and tension (Fig. 3 and 6). Particularly, strains of the order of 500 to 1500 microstrain were found in the cortical shell of vertebral bodies using strain gauges for a $1470 \mathrm{~N}$ compressive force [44] and average compressive and tensile strains (minimum and maximum principal strains) from DIC were found to be -2587 microstrain and 678 microstrain for a compressive force of $2050 \mathrm{~N}$ [14]. These values would have therefore obscured the real nature of internal strain distribution and made impossible to predict where the damage in the vertebral body would initiate. In this context the ability of DVC in identifying internal strain represents an invaluable resource despite its higher strain precision errors at organ level (few hundreds microstrain) [37, 39], when compared 
to DIC (few tens and up to hundred microstrain) [12, 13] or strain gauges (few microstrain) [14].

Another important advantage of DVC relies in its ability to quantify internal microdamage in the bone microstructures. The use of micro-CT image-guided failure assessment $[45,46]$ has allowed three-dimensional analysis of microdamage in bone tissue, allowing the assessment of damage onset and progression under load. In trabecular bone the microdamage is mainly characterized by bending and buckling of the trabeculae at different locations: these phenomena are relatively easy to detect via visual inspection of the sequential micro-CT images during step-wise loading [46, 47]. The use of a specific Matlab script allowed a more precise and reliable coupling of a qualitative assessment of microdamage, to quantitative information about the strain fields (from DVC), throughout the entire volume of the specimens [43]. Interestingly, the use of DVC in vertebral mechanics rarely focused on the coupling of microdamage with strain distribution in the failure region. When this was done, it mainly involved the axial strain [36], which is surely important in a compression loading but provides only incomplete physiological information. Conversely, when the main physiological directions (axial, antero-posterior and lateral-lateral components of strain) were considered, the microdamage development associated to that specific strain condition was not analyzed [34, 35]. Moreover, only scattered information on the average strains at the different levels along the vertebral body are reported [35]. Hussein et al. [35] presented an average compressive strain (minimum principal strain) in six vertebral bodies at three locations; namely superior (-44000 \pm 53000 microstrain), central (-49000 \pm 76000 microstrain) and inferior (-50000 \pm 65000 microstrain) regions. However, no details on the single vertebral bodies were reported and, as indicated by the large scatter in the results, a number of different damage patterns are to be expected. Our findings are in agreement with the results from Hussein et al. [35] where the most important compressive strains were found in caudal direction (or inferior) for both specimen T1 (nearly -76000 microstrain) and specimen T2 (nearly -33000 microstrain). Dissimilarly, the third specimen (T3) experienced highest compressive strains (just above -42000 microstrain) in the cranial region, confirming the high standard deviations reported by [35]. A previous study [28] applied DVC to micro-CT images of porcine trabecular bone during destructive step-wise compression testing. It was found that the axial 
strain values outside the crushed zone ranged from 2000 to -30000 microstrain in the elastic regime and from 2000 to -50000 microstrain during plastic yielding. The latter findings are consistent with the results obtained in this paper where T1 (Fig. 3) experienced local axial strains up to -30000 microstrain in the trabecular bone for the elastic compression step (5\%) and local axial strains up to -60000 microstrain in the trabecular bone for the yield compression step (10\%). The lower axial compressive strains values observed for T2 and T3 (Fig. 4-5) may be probably due to the presence of an external cortical shell in the vertebrae, as well as more complex loading scenarios compared to a compression of a cube of trabecular bone (Gillard et al., 2014).

The current study has some limitations. Firstly, the use of three specimens could not provide enough statistical power to identify consistent trends and investigate the actual mode(s) of failure. However, this sample was sufficient to demonstrate the feasibility of measuring internal strain in the elastic regime, to correlate such elastic strain with the final failure mechanism and to understand the basic strain distribution associated with microdamage in vertebral bodies. A second limitation relates to the use of animal vertebrae (which are certainly different from the human ones [9]). This choice was driven by easier tissue availability compared to human, and by the possibility of fitting the entire vertebral body in the micro-CT scanner and its loading device. Additionally, animal tissue was also used in similar studies [33, 35, 36] and fully justified for explorative in vitro testing of vertebrae [48].

One weak point in the current method is the identification of the linear regime: unfortunately, the test procedure did not allow quantitative assessment of yield in terms of a given offset, as the force-displacement plots are affected by the force drop during the hold phase. However, there was a sharp transition at the end of the linear phase, with an obvious plateau and a decrease in the force-displacement plots, which makes identification of failure quite straightforward.

In the present study we focused only on normal strains, whereas shear strains were not analyzed. This is a reasonable simplification as bone failure is usually associated to a principal strain criterion [21, 49].

It could be interesting to expand this study combining on the same specimen DVC (which provide reliable strain measurements inside the volume of the 
specimen, but not on the surface) and DIC or strain gauges (which provide reliable strain measurements on the surface of the specimen, but cannot interrogate its internal deformation).

\section{Conclusions}

In this paper building up of internal full-field strain from DVC in the elastic regime and progression up to failure was measured in vertebral bodies loaded under step-wise compression loading. Regions of internal microdamage were successfully matched with the distribution of strains, where axial, antero-posterior and laterallateral strains were monitored for all specimens at all levels of compression. The results obtained in this study clearly showed how different vertebral bodies might be subjected to different damage/strain distribution. Thus, consequent microdamage can develop and progress in different ways towards the final failure of the vertebra. Interestingly, DVC-computed strains in the elastic regime had the ability to predict high-strain concentration and therefore damage before failure actually occurred. This has the potential to be implemented in clinical CT assessment of vertebrae, given controlled loading conditions during imaging.

\section{Conflict of interest statement}

None.

\section{Acknowledgements}

The authors would like to thank Remo Antelli for donating the porcine spines, Colin Lupton for technical support in micro-CT maintenance, Dave Hollis (LaVision Ltd) for assistance with DVC software, and Marco Curto for technical support. Funding was provided by the Royal Society (RG130831), University of Portsmouth and European Society of Biomechanics (ESB mobility award 2014). 


\section{REFERENCES}

1. Ferrar L., Jiang G., Adams J. and Eastell R. (2005) Identification of vertebral fractures: An update. Osteoporosis International 16: 717-728.

2. Tancioni F., Lorenzetti M.A., Navarria P., Pessina F., Draghi R., Pedrazzoli P., Scorsetti M., Alloisio M., Santoro A. and Rodriguez y Baena R. (2011) Percutaneous vertebral augmentation in metastatic disease: state of the art. J Support Oncol 9: 4-10.

3. Cristofolini L. (2015) In vitro evidence of the structural optimization of the human skeletal bones. Journal of biomechanics 48: 787-796.

4. Goel V.K., Panjabi M.M., Patwardhan A.G., Dooris A.P. and Serhan H. (2006) Test Protocols for Evaluation of Spinal Implants. Journal of Bone and Joint Surgery 88: 103109.

5. Pollintine P., van Tunen M.S., Luo J., Brown M.D., Dolan P. and Adams M.A. (2010) Time-dependent compressive deformation of the ageing spine: relevance to spinal stenosis. Spine (Phila Pa 1976) 35: 386-394.

6. Brandolini N., Cristofolini L. and Viceconti M. (2014) Experimental Method for the Biomechanical Investigation of Human Spine: a Review Journal Of Mechanics in Medicine and Biology 14: 1430002.

7. Lochmüller E.M., Burklein D., Kuhn V., Glaser C., Muller R., Gluer C.C. and Eckstein F. (2002) Mechanical strength of the thoracolumbar spine in the elderly: prediction from in situ dual-energy X-ray absorptiometry, quantitative computed tomography (QCT), upper and lower limb peripheral QCT, and quantitative ultrasound. Bone 31: 77-84.

8. Singer K., Edmondston S., Day R., Breidahl P. and Price R. (1995) Prediction of thoracic and lumbar vertebral body compressive strength: correlations with bone mineral density and vertebral region. Bone 17: 167-174.

9. Cristofolini L., Brandolini N., Danesi V., Juszczyk M.M., Erani P. and Viceconti M. (2013) Strain distribution in the lumbar vertebrae under different loading configurations. The spine journal : official journal of the North American Spine Society 13: 1281-1292.

10. Freddi A., Olmi G. and Cristofolini L. (2015) Experimental Stress Analysis for Materials and Structures: Stress Analysis Models for Developing Design Methodologies Springer.

11. Ajovalasit A. and Zuccarello B. (2005) Local Reinforcement Effect of a Strain Gauge Installation on Low Modulus Materials. The Journal of Strain Analysis for Engineering Design 40: 643-653.

12. Grassi L. and Isaksson H. (2015) Extracting accurate strain measurements in bone mechanics: A critical review of current methods. J Mech Behav Biomed Mater 50: 43-54.

13. Palanca M., Brugo T.M. and Cristofolini L. (2015) Use of Digital Image Correlation to Understand the Biomechanics of the Vertebra. Journal Of Mechanics in Medicine and Biology 15: 1540004-1540001/1540004-1540010.

14. Gustafson H., Siegmund G. and Cripton P. (2016) Comparison of Strain Rosettes and Digital Image Correlation for Measuring Vertebral Body Strain. Journal of biomechanical engineering Accepted. 
15. Lionello G. and Cristofolini L. (2014) A practical approach to optimizing the preparation of speckle patterns for digital-image correlation. Measurement Science and Technology 25: 107001.

16. Lanyon I.E. (1980) Bone remodelling, mechanical stress, and osteoporosis. In: Osteoporosis, H.F. De Luca (Ed). University Park Press, Baltimore: 129-138.

17. Yang P.F., Bruggemann G.P. and Rittweger J. (2011) What do we currently know from in vivo bone strain measurements in humans? J Musculoskelet Neuronal Interact 11: 820.

18. Fung Y.C. (1980) Bone and cartilage. In: Biomechanics - Mechanical properties of living tissues. Springer Verlag, New York: 383-415.

19. Keaveny T.M., Guo X.E., Wachtel E.F., McMahon T.A. and Hayes W.C. (1994) Trabecular bone exhibits fully linear elastic behavior and yields at low strains. Journal of biomechanics 27: 1127-1136.

20. Kopperdahl D.L. and Keaveny T.M. (1998) Yield strain behavior of trabecular bone. Journal of biomechanics 31: 601-608.

21. Bayraktar H.H., Morgan E.F., Niebur G.L., Morris G.E., Wong E.K. and Keaveny T.M. (2004) Comparison of the elastic and yield properties of human femoral trabecular and cortical bone tissue. Journal of biomechanics 37: 27-35.

22. Cristofolini L. and Viceconti M. (1997) Comparison of Uniaxial and Triaxial Rosette Gages for Strain Measurement in the Femur. Experimental Mechanics 37: 350 - 354.

23. Bergmann G. (2008) Charité Universitaetsmedizin Berlin (2008) “OrthoLoad”. www.orthoload.de, interrogated May 2016. In: Book Charité Universitaetsmedizin Berlin (2008) “OrthoLoad”. www.orthoload.de, interrogated May 2016, Editor (Ed)^(Eds). City.

24. Bouxsein M.L. (2003) Bone quality: where do we go from here? Osteoporos Int 14 Suppl 5: S118-127.

25. Silva M.J., Keaveny T.M. and Hayes W.C. (1997) Load sharing between the shell and centrum in the lumbar vertebral body. Spine (Phila Pa 1976) 22: 140-150.

26. Wang X.-Y., Dai L.-Y., Xu H.-Z. and Chi Y.-L. (2007) The Load-Sharing Classification of Thoracolumbar Fractures: An In Vitro Biomechanical Validation. Spine 32: 121412191210.1097/BRS.1210b1013e318053ec318069.

27. Liu L. and Morgan E.F. (2007) Accuracy and precision of digital volume correlation in quantifying displacements and strains in trabecular bone. Journal of biomechanics 40: 3516-3520.

28. Gillard F., Boardman R., Mavrogordato M., Hollis D., Sinclair I., Pierron F. and Browne M. (2014) The application of digital volume correlation (DVC) to study the microstructural behaviour of trabecular bone during compression. J Mech Behav Biomed Mater 29: 480-499.

29. Dall'Ara E., Barber D. and Viceconti M. (2014) About the inevitable compromise between spatial resolution and accuracy of strain measurement for bone tissue: A 3D zero-strain study. Journal of biomechanics 47: 2956 - 2963. 
30. Roberts B.C., Perilli E. and Reynolds K.J. (2014) Application of the digital volume correlation technique for the measurement of displacement and strain fields in bone: A literature review. Journal of biomechanics 47: 923-934.

31. Palanca M., Tozzi G., Cristofolini L., Viceconti M. and Dall'Ara E. (2015) 3D Local Measurements of Bone Strain and Displacement: Comparison of Three Digital Volume Correlation Approaches. J Biomech Eng (ASME) 137: 071006-071001/071006-071014.

32. Christen D., Levchuk A., Schori S., Schneider P., Boyd S.K. and Muller R. (2012) Deformable image registration and 3D strain mapping for the quantitative assessment of cortical bone microdamage. Journal of Mechanical Behavior of Biomedical Materials 8: 184-193.

33. Hardisty M.R. and Whyne C.M. (2009) Whole bone strain quantification by image registration: a validation study. Journal of biomechanical engineering 131: 064502.

34. Hussein A.I., Barbone P.E. and Morgan E.F. (2012) Digital Volume Correlation for Study of the Mechanics of Whole Bones. Procedia IUTAM 4: 116-125.

35. Hussein A.I., Mason Z.D. and Morgan E.F. (2013) Presence of intervertebral discs alters observed stiffness and failure mechanisms in the vertebra. Journal of biomechanics 46: 1683-1688.

36. Hardisty M., Akens M., Hojjat S., Yee A. and Whyne C. (2012) Quantification of the Effect of Osteolytic Metastases on Bone Strain within Whole Vertebrae Using Image Registration. J Orthop Res 30: 1032-1039.

37. Tozzi G., Dall'Ara E., Palanca M., Curto M., Innocente F. and Cristofolini L. (under review) Strain uncertainties from two digital volume correlation approaches in prophylactically augmented vertebrae: local analysis on bone and cement-bone microstructures. Journal of Mechanical Behavior of Biomedical Materials.

38. Bay B.K., Smith T.S., Fyhrie D.P. and Saad M. (1999) Digital Volume Correaltion: Three-dimensional Strain Mapping Using X-ray Tomography. Experimental Mechanics 39: 217 - 226.

39. Tozzi G., Palanca M., Danesi V., Dall'Ara E. and Cristofolini L. (2015) Strain uncertainties in digital volume correlation of natural and augmented vertebrae. 21st Congress of the European Society of Biomechanics July 5-8 2015, Prague, Czech Republic Book of abstracts, page 192.

40. Danesi V., Zani L., Scheele A., Berra F. and Cristofolini L. (2014) Reproducible reference frame for in vitro testing of the human vertebrae. Journal of biomechanics 47: 313-318.

41. Brear K., Currey J.D., Raines S. and Smith K.J. (1988) Density and temperature effects on some mechanical properties of cancellous bone. Eng Med 17: 163-167.

42. Flay N., Sun W., Brown S., Leach R. and Blumensath T. (2015) Investigation of the Focal Spot Drift in Industrial Cone-beam X-ray Computed Tomography. Digital Industrial Radiology and Computed Tomography (DIR 2015) 22-25 June 2015, Belgium, Ghent - wwwndtnet/appDIR2015.

43. Tozzi G., Zhang Q.H. and Tong J. (2014) Microdamage assessment of bone-cement interfaces under monotonic and cyclic compression. Journal of biomechanics 47: 3466 3474. 
44. Shah J., Hampson W. and Jayson M. (1978) The distribution of surface strain in the cadaveric lumbar spine. J Bone Joint Surg Br 60-B: 246-251.

45. Nazarian A. and Müller R. (2004) Time-lapsed microstructural imaging of bone failure behavior. Journal of biomechanics 37: 55-65.

46. Tozzi G., Zhang Q.H. and Tong J. (2012) 3D real-time micromechanical compressive behaviour of bone-cement interface: experimental and finite element studies. Journal of biomechanics 45: 356-363.

47. Tozzi G., Zhang Q.H., Lupton C., Tong J., Guillen T., Ohrndorf A. and Christ H.J. (2013) Characterisation of a metallic foam-cement composite under selected loading conditions. J Mater Sci Mater Med 24: 2509-2518.

48. Brandolini N., Cristofolini L. and Viceconti M. (2014) Experimental Methods for the biomechanical investigation of the human spine: a review. Journal Of Mechanics in Medicine and Biology 14: 1430002.

49. Helgason B., Perilli E., Schileo E., Taddei F., Brynjolfsson S. and Viceconti M. (2008) Mathematical relationships between bone density and mechanical properties: a literature review. Clinical biomechanics 23: 135-146. 


\section{CAPTIONS}

Fig. 1: Overview of the testing setup: mechanical loading device inside the micro-CT chamber (A). The specimen was potted in PMMA and aligned to the rotation axis of the micro-CT (B). Picture and cross section of a vertebra showing the anatomical directions $(B, C)$. The yellow square in the cross section indicates the final subvolume size (48 voxels, corresponding to approximately $1.862 \mathrm{~mm}, \mathrm{C}$ ). The initial reference state and the compressive steps are shown (D).

Fig. 2: Force-compression curves for the three specimens. The force shows a drop at the end of each step of compression: this corresponds to relaxation while the specimen was allowed to settle (15 minutes) before the micro-CT scan took place (90 minutes). Most of such relaxation took place during the initial 15 minutes. The relaxation during image acquisition (90 minutes) never exceeded 10\% of the initial force magnitude.

Fig. 3: Specimen T1: Internal strain distribution for the three steps of compression. Left: Sagittal micro-CT slice taken at each compression step (the antero (A) and posterior $(P)$ regions are also indicated). The crushed zone of specimen $T 1$ is visible in the images at 10\% and 15\% compression steps (red arrows). The distribution of the Axial, Antero-Posterior and Lateral-Lateral components of strain are plotted over the same sagittal slice in the colored plots. The most strained region corresponded to the damaged area, which gradually progressed in a collapse propagating across the vertebral body, in an approximately transverse plane.

Fig. 4: Specimen T2: Internal strain distribution for the three steps of compression. Left: Sagittal micro-CT slice taken at each compression step (the antero $(A)$ and posterior $(P)$ regions are also indicated). The crushed zone of specimen $T 2$ is visible in the images at 10\% and 15\% compression steps (red arrows). The distribution of the Axial, Antero-Posterior and Lateral-Lateral components of strain are plotted over the same sagittal slice in the colored plots. The most strained region corresponded to the damaged area, which gradually progressed in a collapse propagating across the vertebral body, in an approximately caudal-cranial direction. 
Fig. 5: Specimen T3: Internal strain distribution for the three steps of compression. Left: Sagittal micro-CT slice taken at each compression step (the antero (A) and posterior $(P)$ regions are also indicated). The crushed zone of specimen T3 is visible in the images at 10\% and 15\% compression steps (red arrows). The distribution of the Axial, Antero-Posterior and Lateral-Lateral components of strain are plotted over the same sagittal slice in the colored plots. The most strained region corresponded to the damaged area, which gradually progressed in a collapse propagating across the vertebral body, in an approximately transverse plane.

Fig. 6: The averages were computed within each transverse slice for the Axial, Antero-Posterior and Lateral-Lateral components of strain. The plots show the trend of such average strains along the vertebra (caudal-cranial direction), and their progression as compression increased (5\%,10\% and 15\% steps, for the three specimens). In general, an incremental strain pattern among the consecutive compression steps was observed in all specimens (T1, T2 and T3). The slices where the largest strains were observed corresponded to the areas where collapse was localized (Fig. 3-5). 
Fig 1
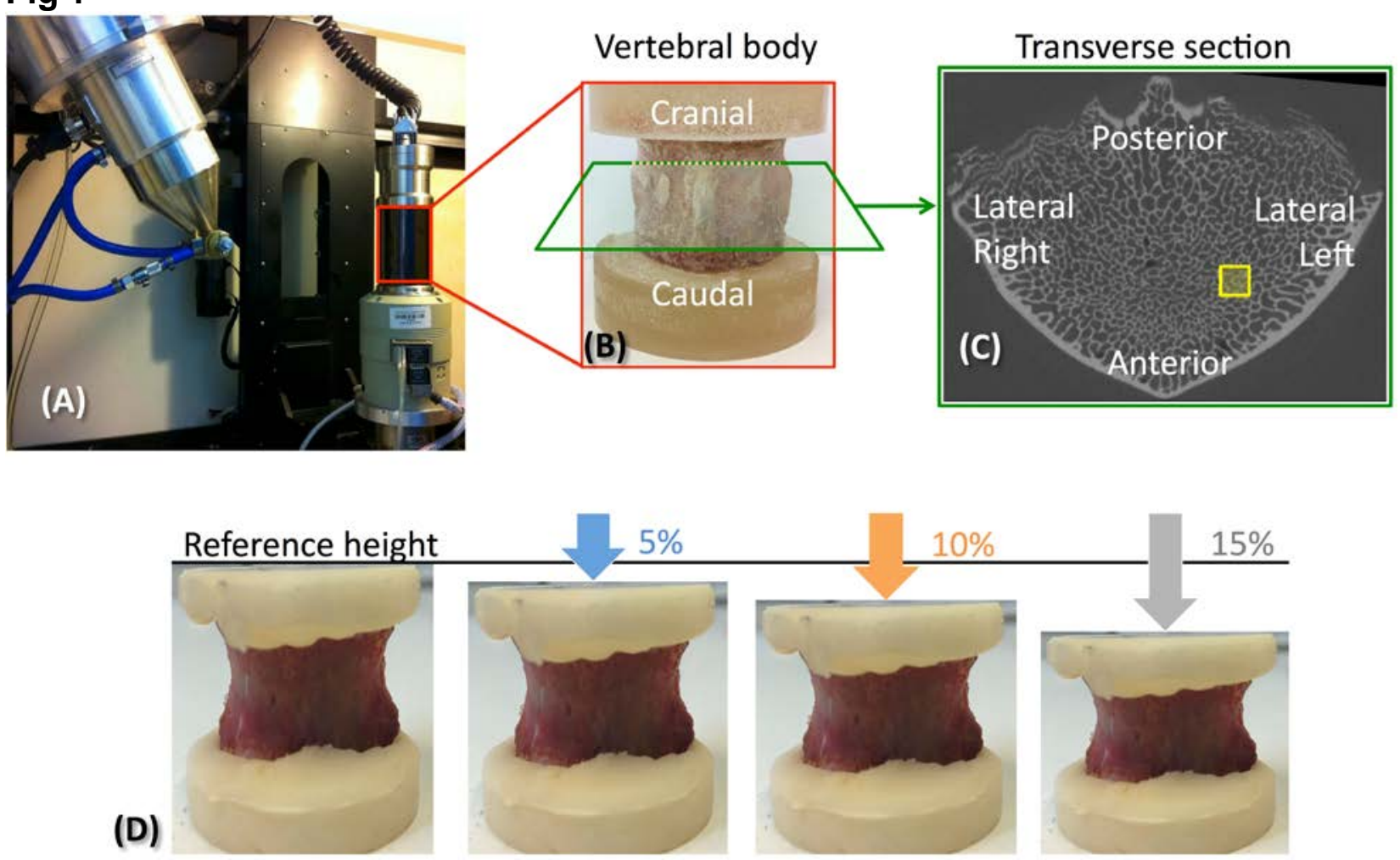

Fig 2

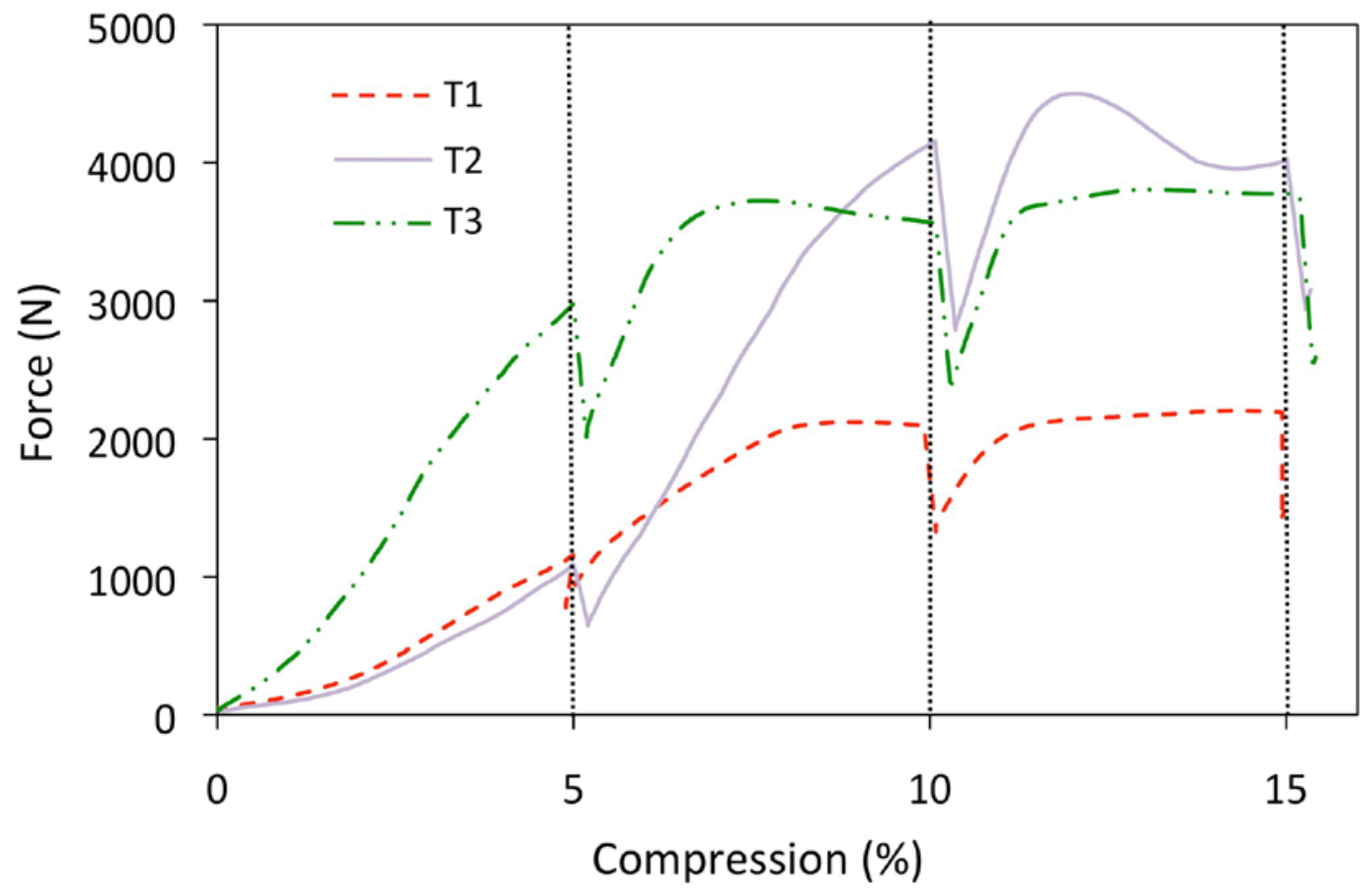


Fig 3

Slice

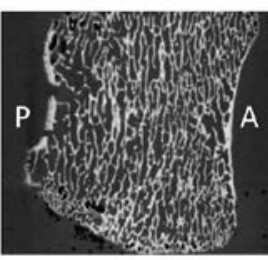

$10 \%$

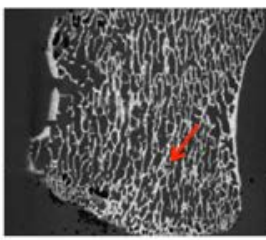

$15 \%$

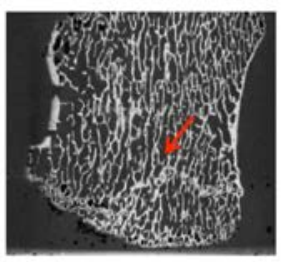

Axial Strain

(microstrain)
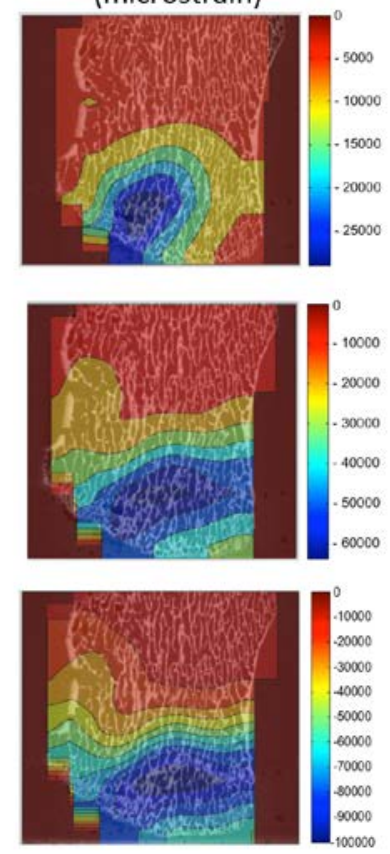

Antero-Posterior Strain (microstrain)
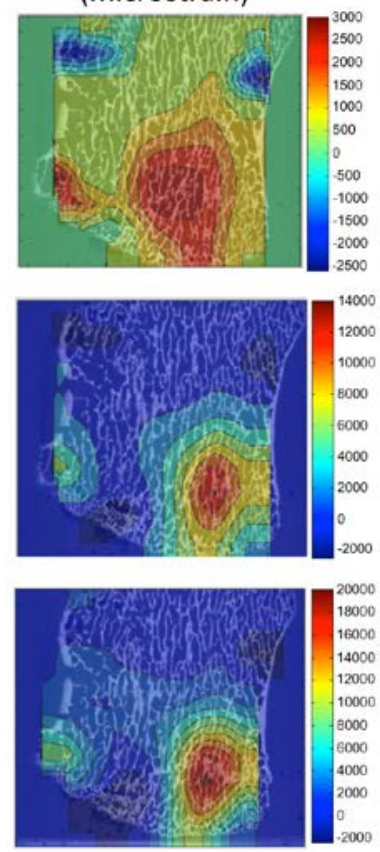

Lateral-Lateral Strain (microstrain)
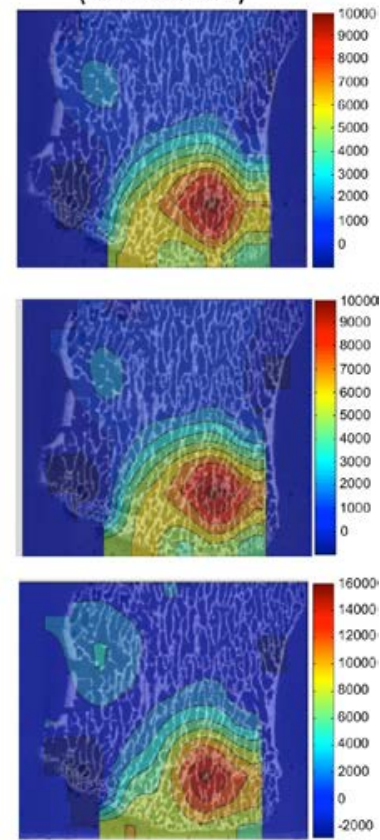

Fig 4
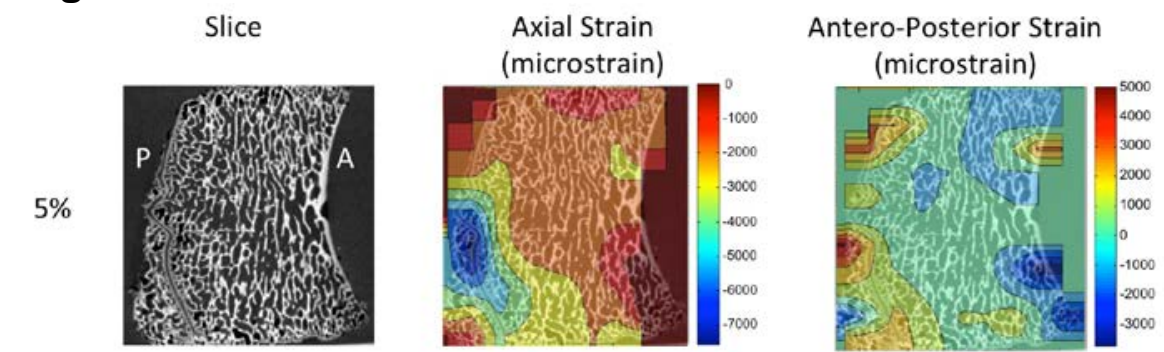

Lateral-Lateral Strain
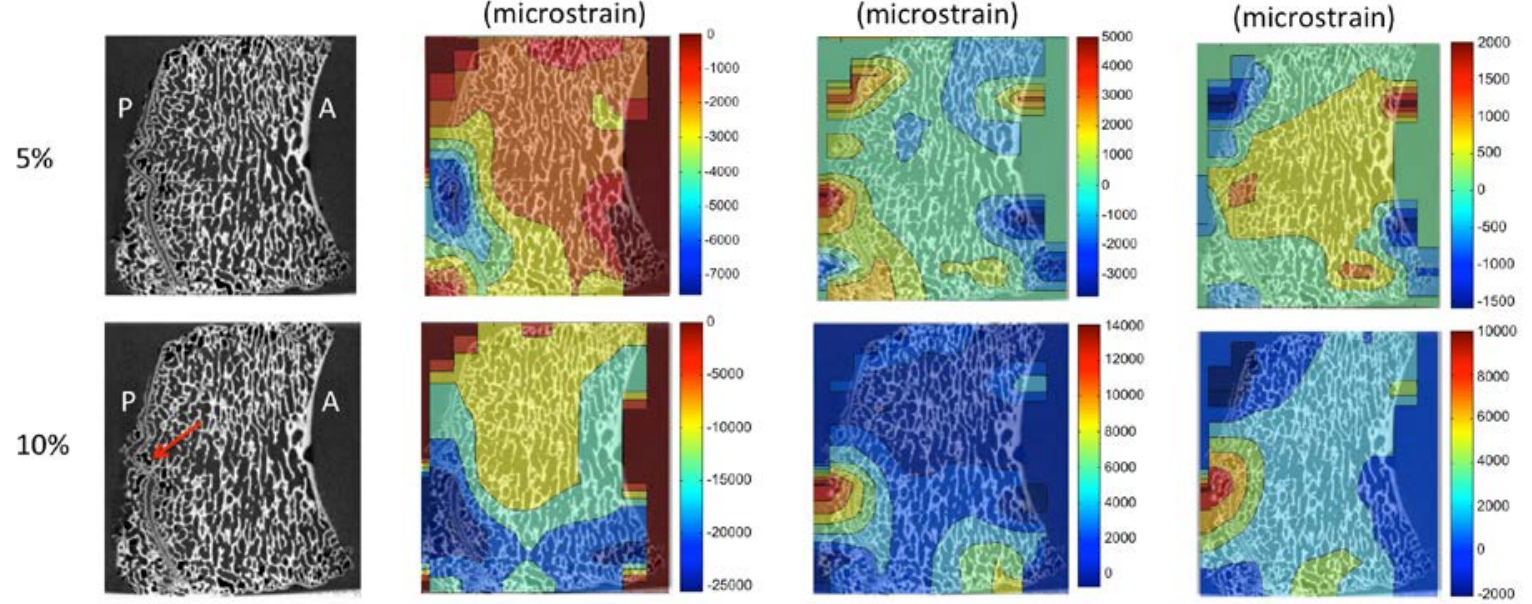

$15 \%$
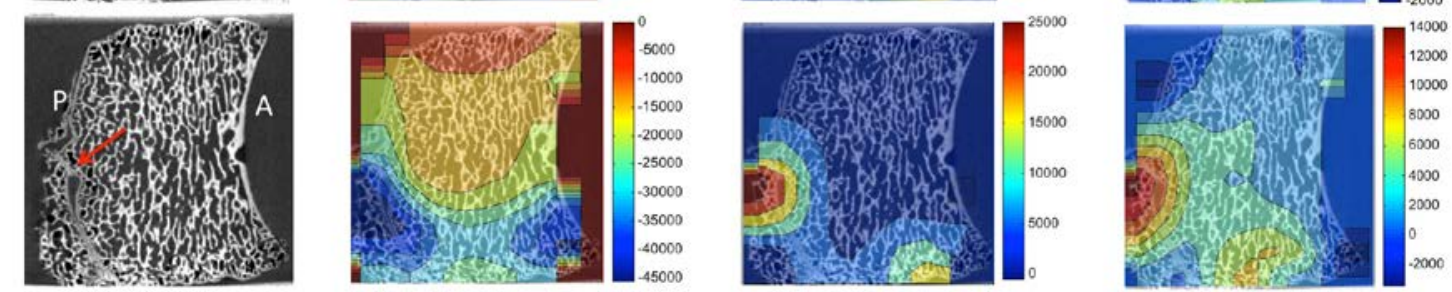
Fig 5

Slice
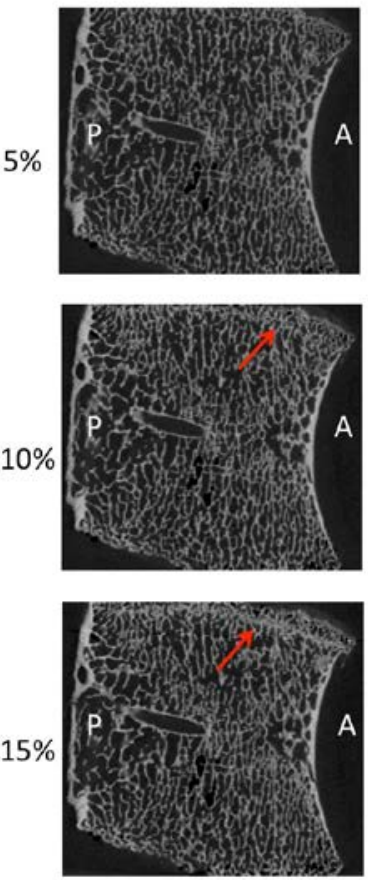

Axial Strain

(microstrain)
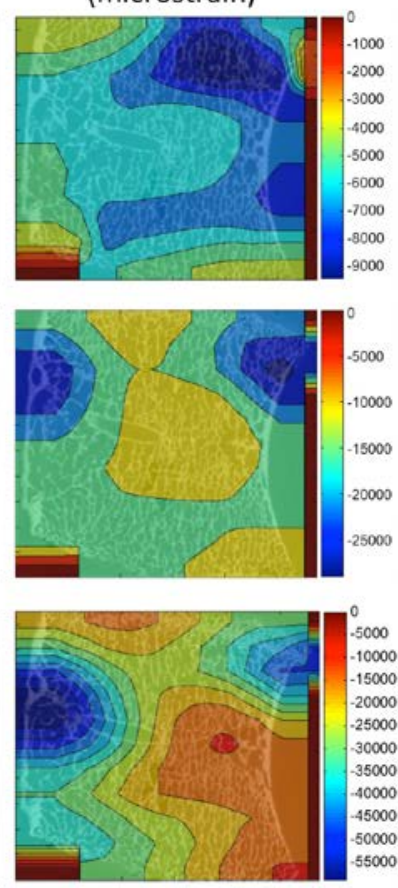

Antero-Posterior Strain (microstrain)
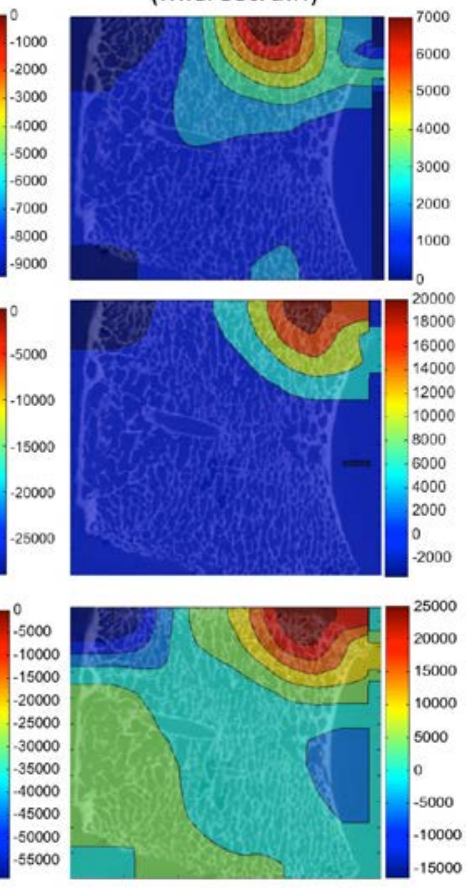

Lateral-Lateral Strain (microstrain)
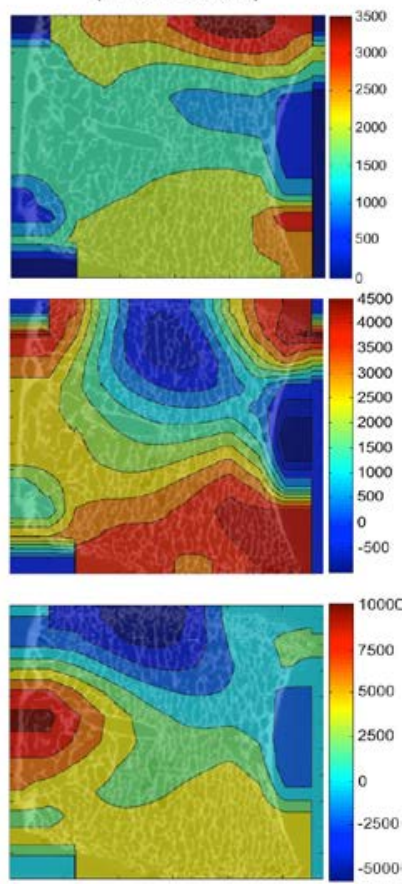

Fig 6

Compressive steps:
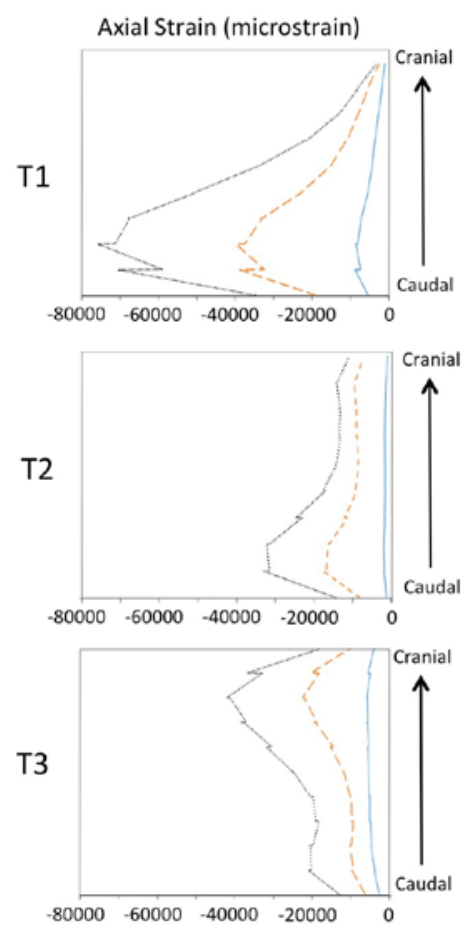

$--10 \%$
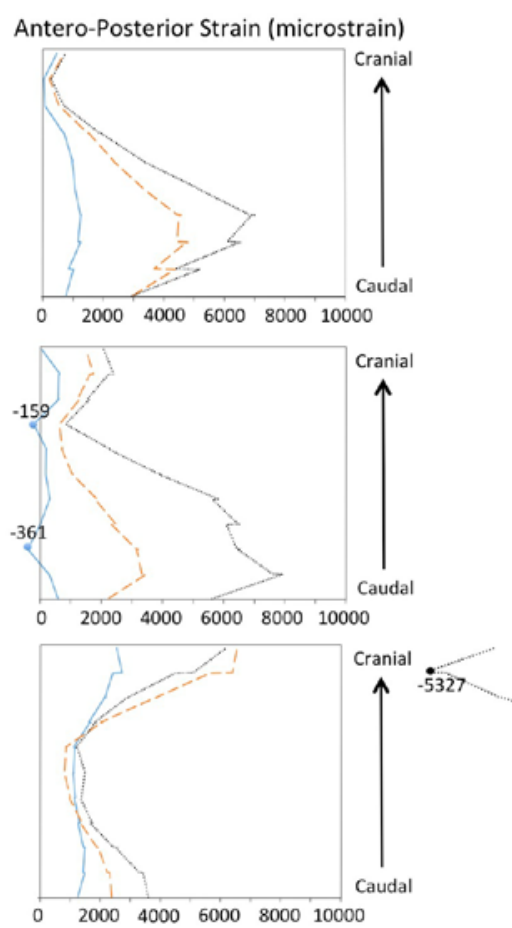

$15 \%$

Lateral-Lateral Strain (microstrain)
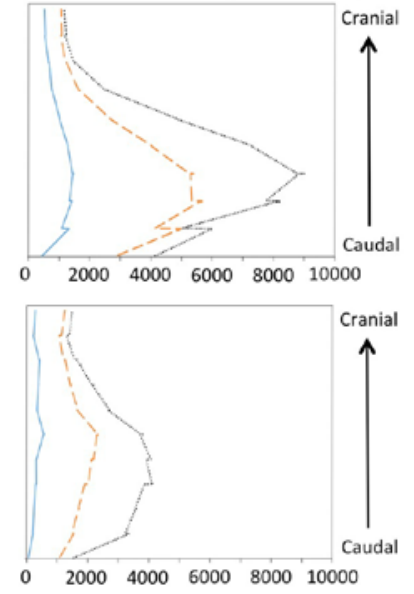

$0 \quad 2000 \quad 4000 \quad 6000 \quad 8000 \quad 10000$

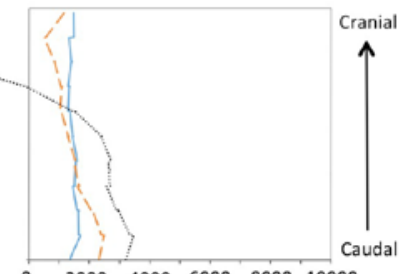

